\title{
La ablación con catéter no redujo la mortalidad o el riesgo de desenlaces cardiovasculares, pero si condujo a mejorías en la calidad de vida de los pacientes con fibrilación auricular
}

Catheter ablation did not reduce mortality or risk of cardiovascular events but did lead to improvement in quality of life in patients with atrial fibrillation

\section{Comentado de:}

Packer DL, et al. JAMA 2019;321(13):1261-12741

Mark DB, et al. JAMA 2019;321(13):1275-1285²

\section{Importancia}

La ablación con catéter es eficaz para restablecer el ritmo sinusal en la fibrilación auricular (FA), pero sus efectos sobre la mortalidad y el riesgo de accidente cerebrovascular, así como, sus beneficios en la calidad de vida a largo plazo, son inciertos.

\section{Objetivos}

Este estudio evaluó si la ablación con catéter es más efectiva que la terapia médica convencional para mejorar estos resultados en pacientes con FA.

\section{Diseño, ámbito y participantes}

Ensayo clínico aleatorizado abierto, multicéntrico, involucrando 126 centros en 10 países. Se incluyeron un total de 2.204 pacientes sintomáticos con FA mayores de 65 años, o menores de 65 años con uno o más factores de riesgo de accidente cerebrovascular (ACV), que tuvieran dos o más episodios de FA paroxística o un episodio de FA persistente en los seis meses anteriores; enrolados entre noviembre de 2009 y abril de 2016, con seguimiento hasta el 31 de diciembre de 2017.

\section{Intervenciones}

Los pacientes del grupo ablación con catéter $(n=1108)$ recibieron aislamiento de la vena pulmonar, acompañado de procedimientos de ablación adicionales de acuerdo al criterio de los investigadores. El grupo tratamiento farmacológico $(n=1096)$ recibió terapia convencional para control del ritmo y/o la frecuencia cardíaca, guiada por las guías de práctica clínica contemporáneas.

\section{Medidas de desenlace principales}

El resultado primario fue un compuesto de muerte, apoplejía, sangrado grave o paro cardíaco. Los resultados secundarios incluyeron, entre otros: mortalidad por todas las causas; mortalidad total u hospitalización cardiovascular; y recurrencia de la FA.

Los puntos finales pre-especificados de calidad de vida coprimarios a los 12 meses, incluyeron el puntaje sumario del efecto de fibrilación auricular en la calidad de vida (AFEQT, por sus iniciales en inglés) (rango 0 a 100; en el que 0 indica una discapacidad completa y 100 indica que no hay discapacidad, y la diferencia clínicamente importante a nivel del paciente es de 5 puntos) y los puntajes de frecuencia y severidad del inventario de síntomas específicos de Mayo AF (MAFSI, por sus iniciales en inglés) (puntaje de frecuencia: rango 0 a 40, en el que 0 indica que no hay síntomas y 40 indica síntomas más graves, y la diferencia clínicamente importante a nivel del paciente es $-1,6$ puntos; puntaje de gravedad: rango, $0 a 30$, en el que 0 indica que no hay síntomas y 30 indica síntomas más graves, y la diferencia clínicamente importante a nivel del paciente es $-1,3$ puntos).

\section{Resultados}

De los 2.204 pacientes (mediana de edad, 68 años; 1.385 pacientes [63\%] eran hombres, 946 [43\%] tenían FA paroxístiva, and 1256 [57\%] tenían FA persistente), la mediana de seguimiento fue 48,5 meses, y 1.968 (89,3\%) completaron el estudio. De los pacientes asignados a la ablación con catéter, 1.006 (90,8\%) recibieron el procedimiento. De los pacientes asignados a la terapia con medicamentos, $301(27,5 \%)$ finalmente recibieron ablación con catéter.

En el análisis por intención de tratar, el resultado primario combinado ocurrió en $8,0 \%(n=89)$ de los pacientes en el grupo de ablación frente al 9,2\% $(n=101)$ de los pacientes en el grupo de tratamiento farmacológico (Hazard ratio - HR - 0,86; intervalo de confianza - IC - $95 \% 0,65$ a 1,15, p=0,30).

No hubo diferencia en la mortalidad por todas las causas $(5,2$ versus $6,1 \%$, HR 0,85; IC95\% 0,60 a 1,21). La muerte o la hospitalización por causa cardiovascular ocurrió con menos frecuencia con la ablación con catéter 51,7 vs $58,1 \%$; HR 0,83 (IC $95 \%$ 0,74 a 0,93 ), al igual que la tasa de recurrencia de FA 49,9 vs $69,5 \%$; HR 0,52 (IC $95 \% 0,45$ a 0,60).

La media del puntaje sumario del AFEQT fue más favorable en el grupo ablación con catéter que en el grupo de terapia farmacológica a los 12 meses $(86,4$ puntos vs 80,9 puntos) (diferencia ajustada, 5,3 puntos [IC $95 \%, 3,7$ a 6,9$]$; $p<0,001$ ). Los puntajes de frecuencia y gravedad de MAFSI también fueron más favorables en el grupo de ablación con catéter que en el grupo de tratamiento farmacológico a los 12 meses con medias de 6,4 puntos frente a 8.1 puntos (diferencia ajustada, -1.7 puntos, IC $95 \%$, $-2,3$ a $-1,2 ; p<0,001$ ), y 5,0 puntos frente a 6,5 puntos (diferencia ajustada, $-1,5$ puntos, IC $95 \%,-2,0$ a $-1,1 ; p<0.001)$, respectivamente.

\section{Conclusiones}

Entre los pacientes con FA, la estrategia de la ablación con catéter, en comparación con la terapia médica, no redujo significativamente el resultado primario compuesto; sin embargo el efecto estimado se vio afectado por tasas de eventos más bajos de lo esperado y cruces de tratamiento.

La ablación condujo a beneficios clínicamente importantes en la calidad de vida a los 12 meses. Estos hallazgos pueden ayudar a guiar las decisiones en el manejo de la fibrilación auricular.

Conflicto de interés de los autores: Los autores reportaron sus conflictos de interés. El estudio fue financiado por organismos públicos y privados (National Heart, Lung, and Blood Institute, St Jude Foundation and Corporation, BiosenseWebster Inc, Medtronic Inc, Boston Scientific Corporation, y Mayo Clinic). 


\section{Comentario}

En los pacientes con FA, los tres objetivos principales del tratamiento son la reducción del riesgo de ACV, la prevención de la miocardiopatía mediada por taquicardia y el alivio de los síntomas. Para lograr el primer objetivo, la mayoría de los pacientes con FA requiere anticoagulación oral. Con respecto a los otros dos objetivos, se pueden lograr con fármacos que controlen el ritmo o la frecuencia cardíaca y la ablación por catéter (AC).

La AC para el tratamiento de la FA se introdujo clínicamente en la década de 1990 como terapia de último recurso para los pacientes con enfermedad sintomática refractaria al tratamiento farmacológico ${ }^{3,4}$. Sin embargo, durante los siguientes 20 años, la ablación se convirtió en una opción terapéutica para un espectro más amplio de pacientes con FA. Esta evolución se debió a varios factores, incluyendo el logro de altas tasas de remisión exitosa de la FA.

Si bien los primeros informes especularon que el procedimiento podría ser curativo ${ }^{3,5,6}$, experiencias adicionales demostraron que una minoría de pacientes sometidos a ablación experimentó FA recurrente si el seguimiento era lo suficientemente prolongado. A partir de allí, la atención se centró en los beneficios de la ablación en la calidad de vida ${ }^{7}$.

La calidad de vida en la FA sintomática es similar a la de un paciente que ha sufrido un infarto y ha sobrevivido, e incluso los pacientes con FA asintomática padecen deterioro en su calidad de vida, ya sea por el recuerdo de la arritmia, la preocupación por su posible reaparición o la limitación en sus actividades ${ }^{8}$.

En el estudio resumido, los desenlaces secundarios mortalidad cardiovascular y hospitalización por causa cardiovascular, al igual que la tasa de recurrencia de FA, fueron menos frecuentes en pacientes que recibieron AC. Este último hallazgo coincide con estudios previos que demostraron tasas de recurrencias más bajas en este grupo de pacientes ${ }^{9,10}$.

La mejoría en la calidad de vida del grupo de AC fue significativamente mayor que en el grupo de tratamiento farmacológico, y los beneficios de la ablación tendieron a ser máximos a los 12 meses. Resultados similares se encontraron en el estudio "The CAPTAF Randomized Clinical Trial"11.

Entre las limitaciones del estudio se encuentran la falta de cegamiento de los grupos, las pérdidas de pacientes y los cruces de tratamiento. Comparaciones de los resultados en el análisis por intención de tratar con el análisis por protocolo según el tratamiento recibido sugieren que el efecto combinado de cruces y pérdidas redujeron el efecto estimado del tratamiento y su precisión.

\section{Conclusiones del comentador}

Teniendo en cuenta que la FA es la arritmia más frecuente en la población general con repercusión clínica, que genera el mayor número de consultas a los servicios de urgencias con más días de ingreso hospitalario ${ }^{12} \mathrm{y}$, además, que su prevalencia e incidencia se han incrementado con el tiempo, ya sea por el envejecimiento de la población, el aumento de los factores de riesgo cardiovascular o la mejora de la supervivencia a enfermedades cardiovasculares, resulta razonable pensar en la opción de tratamiento que logre mejorar la calidad de vida a través de reducir síntomas, complicaciones y recurrencias de la FA, adecuando el tratamiento a las preferencias y posibilidades de cada paciente.

Yanina Avendaño [ Servicio de Medicina Familiar y Comunitaria, Hospital Italiano de Buenos Aires yanina.avendano@hospitalitaliano.org.ar ]

Avendaño Y. La ablación con catéter no redujo la mortalidad o el riesgo de desenlaces cardiovasculares, pero si condujo a mejorías en la calidad de vida de los pacientes con fibrilación auricular . Evid Actual Pract Ambul. 2019;22(1):e001991. Comentado de: Packer DL, et al. Effect of Catheter Ablation vs Antiarrhythmic Drug Therapy on Mortality, Stroke, Bleeding, and Cardiac Arrest Among Patients With Atrial Fibrillation. JAMA 2019;321(13):1261-1274. PMID 30874766; y Mark DB, et al. Effect of Catheter Ablation vs Medical Therapy on Quality of Life Among Patients With Atrial Fibrillation The CABANA Randomized Clinical Trial. JAMA 2019;321(13):1275-1285. PMID 30874716

\section{Referencias}

1. Packer DL, Mark DB, Robb RA, et al. Effect of Catheter Ablation vs Antiarrhythmic Drug Therapy on Mortality, Stroke, Bleeding, and Cardiac Arrest Among Patients With Atrial Fibrillation. JAMA. 2019;321(13):1261-1274. Available from: doi:10.1001/jama.2019.0693.

2. Mark DB, Anstrom KJ, Sheng S, et al. Effect of Catheter Ablation vs Medical Therapy on Quality of Life Among Patients With Atrial Fibrillation The CABANA Randomized Clinical Trial. 2019;321(13):1275-1285. Available from: doi:10.1001/jama.2019.0692.

3. Jaïs $P$, Haïssaguerre M, Shah DC, Chouairi S, Gencel L, Hocini M, et al. A focal source of atrial fibrillation treated by discrete radiofrequency ablation. Circulation. 1997;95(3):572-576. Available from: 10.1161/01.cir.95.3.572.

4. HAÏSSAGUERRE M, GENCEL L, FISCHER B, MÉTAYER P, POQUET F, MARCUS FI, et al. Successful Catheter Ablation of Atrial Fibrillation. Journal of Cardiovascular Electrophysiology. 1994;5(12):1045-1052. Available from: 10.1111/j.1540-8167.1994.tb01146.x.

5. Wazni OM, Marrouche NF, Martin DO, Verma A, Bhargava M, Saliba W, et al. Radiofrequency ablation vs antiarrhythmic drugs as first-line treatment of symptomatic atrial fibrillation: a randomized trial. JAMA. 2005;293(21):2634-40. Available from: 10.1001/jama.293.21.2634.

6. Pappone C, Augello G, Sala S, Gugliotta F, Vicedomini G, Gulletta S, et al. A randomized trial of circumferential pulmonary vein ablation versus antiarrhythmic drug therapy in paroxysmal atrial fibrillation: the APAF Study. Journal of the American College of Cardiology. 2006 dec;48(11):23407. Available from: 10.1016/j.jacc.2006.08.037.

7. Wokhlu A, Monahan KH, Hodge DO, Asirvatham SJ, Friedman PA, Munger TM, et al. Long-term quality of life after ablation of atrial fibrillation the impact of recurrence, symptom relief, and placebo effect. Journal of the American College of Cardiology. 2010 may;55(21):2308-16. Available from: 10.1016/j.jacc.2010.01.040.

8. Dorian P, Jung W, Newman D, Paquette M, Wood K, Ayers GM, et al. The impairment of health-related quality of life in patients with intermittent atrial fibrillation: implications for the assessment of investigational therapy. Journal of the American College of Cardiology. 2000;36(4):1303-1309. Available from: 10.1016/S0735-1097(00)00886-X.

9. Noheria A, Kumar A, Wylie JV, Josephson ME. Catheter ablation vs antiarrhythmic drug therapy for atrial fibrillation: a systematic review. Archives of internal medicine. 2008 mar;168(6):581-6. Available from: 10.1001/archinte.168.6.581. 
10. Wilber DJ, Pappone C, Neuzil P, Paola AD, Marchlinski F, Natale A, et al. Comparison of antiarrhythmic drug therapy and radiofrequency catheter ablation in patients with paroxysmal atrial fibrillation: a randomized controlled trial. JAMA. 2010 jan;303(4):333-40. Available from: 10.1001/jama. 2009.2029.

11. Blomström-Lundqvist C, Gizurarson S, Schwieler J, Jensen SM, Bergfeldt L, Kennebäck G, et al. Effect of Catheter Ablation vs Antiarrhythmic Medication on Quality of Life in Patients With Atrial Fibrillation: The CAPTAF Randomized Clinical Trial. JAMA. 2019 mar;321(11):1059-1068. Available from: 10.1001/jama.2019.0335.

12. Kannel WB, Wolf PA, Benjamin EJ, Levy D. Prevalence, incidence, prognosis, and predisposing conditions for atrial fibrillation: population-based estimates. The American Journal of Cardiology. 1998;82(7):2-9. Available from: 10.1016/S0002-9149(98)00583-9. 